\title{
ANALYTICAL REPRESENTATION OF INTEGRALS RELEVANT TO COHERENT IMAGING*
}

\author{
BY \\ J. M. PIMBLEY (Rensselaer Polytechnic Institute, Troy, New York) \\ AND \\ S. D. SILVERSTEIN (General Electric, Schenectady, New York)
}

\begin{abstract}
This work presents and evaluates three classes of definite integrals which can arise in the signal processing analysis of coherent imaging systems. All integrals yield to the method of contour integration in the complex plane. Such a treatment requires physically reasonable constraints on the beam divergence, apodizing function of the phased array. The physical foundation of the three integral classes is described in the text.
\end{abstract}

I. Introduction. Theoretical research in applied fields such as signal analysis and signal processing often leads to mathematical relations which do not have readily accessible analytical solutions. Of particular interest to this work are classes of integral relations which are neither documented in any of the well-known large integral tables nor generated by the powerful mathematical computer expert system commonly known by the program name MACSYMA. Fast computers have provided workers in a variety of theoretical sciences with numerical solution paths. Numerical solutions are, of course, very important and extremely useful. However, if analytical solutions can be found they possess much more flexibility to the variation of intrinsic parameters, and allow the worker to develop more of an intuitive-physical insight into the parametric nature of the solutions to physical problems.

In this note we present and evaluate three classes of definite integrals which have their physical origins in the signal analysis fields relevant to coherent imaging systems. Such imaging systems can include, for example, radar, sonar, ultrasound, and radioastronomy imaging systems. We have added an appendix to the text in which we discuss a specific example of the physical basis for these integrals. For further reading concerning the physical basis for some of the integrals described herein, the reader is referred to some of the published papers by one of the authors [1-4].

${ }^{*}$ Received March 28, 1988.

(C)1989 Brown University 
II. First class of integrals. Of the three classes of integrals described in the introduction, we begin with $\Psi_{m}^{N}(x)$ :

$$
\Psi_{m}^{N}(x)=\int_{-\infty}^{\infty} d z A(z) e^{i 2 m z x} \frac{\sin ^{2 m} z}{\sin ^{2 m}(z / N)} .
$$

The evaluation of this integral, as well as all others in this communication, proceeds with the method of contour integration in the complex plane, see for example, [5-6]. As is well known, contour integration implies the conversion of a definite integral to a suitably chosen closed-contour integral in the complex plane. An integral about a closed contour is proportional to the sum of the residues of the integrand enclosed by the contours. A "suitably chosen" contour is a contour for which the integrals over all constituent segments are known or expressible in terms of the definite integral to be evaluated.

The function $A(z)$ of (1) models the apodizing function for a detector phased array system. This identification implies several restrictions. Along the real axis, we expect to find a smooth (i.e., nonsingular), even function that attains a maximum value at the origin and decays to zero at infinity. Our contour integration approach will necessitate strengthening this constraint such that $A(z)$ decays to zero as $z$ goes to infinity everywhere in the complex plane (i.e., imaginary axis as well as real axis). Furthermore, while singularities of $A(z)$ are allowed at points not on the real axis, we permit only simple poles for these singularities. A typical example of an allowable $A(z)$ is $1 /\left(1+\beta^{2} z^{2}\right)$. This Lorentzian function possesses simple poles at $i / \beta$ and $-i / \beta$.

We consider two possible contours of integration, $C_{1}$ and $C_{2}$, in our computations. Both contours coincide with the real axis from $-R$ to $+R$. To $C_{1}$ is then added a semicircular component of radius $R$ in the upper (positive imaginary) half-plane. Similarly, $C_{2}$ possesses an additional semicircular component of radius $R$ in the lower (negative imaginary) half-plane. Both contours are closed. As $R$ goes to infinity, $C_{1}$ will enclose the entire upper half-plane while $C_{2}$ encloses the lower half-plane.

The integrand of (1) has no poles on the real axis, and thus no residues there, as the $\sin ^{2 m} z$ numerator vanishes and maintains a finite limit when the denominator vanishes at $z=j N \pi$ for all integers $j$. If $x \geq 1$, then, one may integrate over the contour $C_{1}$ since the term $e^{i 2 m z x}$ in the integrand of (1) will force a zero contribution from the upper half-plane semicircle contour integral as the contour radius goes to infinity. This zero contribution implies $\Psi_{m}^{N}(x)$ is equal to $2 \pi i$ times the sum of the residues of the integrand of equation (1) in the upper-half-plane. All such residues emanate from simple poles of $A(z)$. Similarly, when $x \leq-1$, the contour $C_{2}$ is appropriate since the exponential term renders the integrand negligible along the lower half-plane semicircle (at infinite radius). Thus, $\Psi_{m}^{N}(x)$ is given by $-2 \pi i$ times the sum of the residues of the integrand in the lower-half-plane. If $A(z)$ is the Lorentzian $1 /\left(1+\beta^{2} z^{2}\right)$, then

$$
\Psi_{m}^{N}(x)=\frac{\pi}{\beta} e^{-2 m x / \beta}\left[\frac{\sinh (1 / \beta)}{\sinh (1 /(\beta N))}\right]^{2 m} \text { for } x \geq 1 .
$$


Replacing $x$ by $-x$ in the above expression yields the result for $x \leq-1$. Note that in both cases $\Psi_{m}^{N}(x)$ goes to zero as the Lorentzian parameter $\beta$ goes to zero.

The results of the preceding paragraph originate from the observation that the choice of either $C_{1}$ (for $x \geq 1$ ) or $C_{2}$ (for $x \leq-1$ ) allowed us to invoke Cauchy's theorem [5] for the integral about a closed contour of an analytic function in the complex plane. With $x$ in the interval $(-1,1)$, however, the situation is not so simple. In this case the integrand diverges along infinite semicircles in both the upper and lower half-planes. Thus, neither $C_{1}$ nor $C_{2}$ is an appropriate contour of integration. We resolve this difficulty by decomposing the numerator $\sin ^{2 m} z$ as

$$
\sin ^{2 m} z=\left[\sin ^{2 m} z-f_{q}(z)\right]+f_{q}(z),
$$

where

$$
f_{q}(z)=\left(\frac{-1}{4}\right)^{m} \sum_{k=0}^{q}(-1)^{k}\left(\begin{array}{c}
2 m \\
k
\end{array}\right) e^{-i 2 z(m-k)} .
$$

We construct the function $f_{q}(z)$ of Eq. (2b) by expressing $\sin z$ as $\left(e^{i z}-e^{-i z}\right) /(2 i)$ and expanding this quantity raised to the $2 m$ power. The bracketed quantity $\left[\sin ^{2 m} z-\right.$ $f_{q}(z)$ ] of Eq. (2a) now decays to zero along the semicircular portion of the contour $C_{1}$ while $f_{q}(z)$ vanishes along the semicircular portion of contour $C_{2}$ (for $x \geq 0$ ). Thus, we evaluate $\Psi_{m}^{N}(x)$ of equation (1) by evaluating two integrals generated by this decomposition of $\sin ^{2 m} z$. We must add that the integer $q$ may vary from 0 to $m-1$ and is completely determined by the value of the independent variable $x$ through the requirement

$$
\frac{m-q-1}{m}<x<\frac{m-q}{m}, \quad q=0,1,2, \ldots, m-1 .
$$

Analogous results apply to the case $-1<x<0$.

The decomposition of the numerator $\sin ^{2 m} z$ effectively turns the isolated real points $z=j N \pi$ into singularities. Thus, evaluation of the two separate integrals will require computation of residues at these singularities. We now modify our contour definitions somewhat so that $C_{1}$ "jumps" over each singularity via semicircular excursions into the positive half-plane while $C_{2}$ avoids the singularities with negative half-plane semicircles. From Eq. (1) and the ensuing discussion on the integrand decomposition, we write

$$
\Psi_{m}^{N}(x)=\int_{-\infty}^{\infty} d z A(z) e^{i 2 m z x} \frac{\sin ^{2 m} z-f_{q}(z)}{\sin ^{2 m}(z / N)}+\int_{-\infty}^{\infty} d z A(z) e^{i 2 m z x} \frac{f_{q}(z)}{\sin ^{2 m}(z / N)} .
$$

Evaluating the first integral over $C_{1}$ and the second over $C_{2}$, we write

$$
\begin{aligned}
\Psi_{m}^{N}(x)=-2 \pi i \sum_{n=-\infty}^{\infty} \text { Residue } & \left\{A(z) e^{i 2 m z x} \frac{f_{q}(z)}{\sin ^{2 m}(z / N)}\right\} \\
+2 \pi i[\text { UHP Residues } & \left\{A(z) e^{i 2 m z x} \frac{\sin ^{2 m} z-f_{q}(z)}{\sin ^{2 m}(z / N)}\right\} \\
& \text {-LHP Residues } \left.\left\{A(z) e^{i 2 m z x} \frac{f_{q}(z)}{\sin ^{2 m}(z / N)}\right\}\right] .
\end{aligned}
$$


The summation on $n$ simply counts the singularities along the real axis while the last bracketed term specifies the sum of all upper-half-plane (UHP) residues minus the sum of all lower-half-plane (LHP) residues. These latter residues exist only due to the simple poles of $A(z)$.

To proceed from Eq. (5) we must construct the residue of the quantity within the curly brackets. This task requires a Taylor series expansion of $[\sin (z / N)]^{-2 m}$ about the singularity $z=n N \pi$. We find

$$
(\sin u)^{-2 m}=(u-n \pi)^{-2 m}\left\{1+\sum_{r=1}^{\infty} \xi_{r, m}(u-n \pi)^{2 r}\right\},
$$

where

$$
\begin{gathered}
\xi_{r, m}=\sum_{t=1}^{r} c_{r t}\left(\begin{array}{c}
2 m+t-1 \\
t
\end{array}\right), \quad \text { for } r \geq 1, \\
c_{r t}=(-1)^{r-t} \sum_{i_{1}=0}^{r-t} \frac{1}{\left(2 i_{1}+3\right) !} \sum_{i_{2}=0}^{r-t-i_{1}} \frac{1}{\left(2 i_{2}+3\right) !} \times \cdots \\
\times \sum_{i_{t-1}=0}^{r-t-i_{1}-\cdots-i_{t-2}} \frac{1}{\left(2 i_{t-1}+3\right) !} \frac{1}{\left[2\left(r-t-i_{1}-i_{2}-\cdots-i_{t-1}\right)+3\right] !} .
\end{gathered}
$$

To Eq. (6b) we add the definition $\xi_{0, m}=1$.

We must also expand the product $A(z) f_{q}(z) e^{i 2 m z x}$ in a Taylor series for the eventual goal of determining the complete residue. With the observation that

$$
\begin{gathered}
\frac{d^{2 s-1}\left[A(z) f_{q}(z) e^{i 2 m z x}\right]}{d z^{2 s-1}}=\left(\frac{-1}{4}\right)^{m} e^{i 2 m n \pi N x} \sum_{j=0}^{2 s-1}\left(\begin{array}{c}
2 s-1 \\
j
\end{array}\right) \frac{d^{j} A(n \pi N)}{d z^{j}}(-2 i)^{2 s-1-j} \\
\times \sum_{j=0}^{2 s-1}\left(\begin{array}{c}
2 s-1 \\
j
\end{array}\right) \frac{d^{j} A(n \pi N)}{d z^{j}}(-2 i)^{2 s-1-j} \sum_{k=0}^{q}(-1)^{k}\left(\begin{array}{c}
2 m \\
k
\end{array}\right)(m-m x-k)^{2 s-1-j},
\end{gathered}
$$

we may merge all preliminary results. From Eqs. (5-7), we find

$$
\begin{aligned}
& \Psi_{m}^{N}(x)= \frac{(-1)^{m} \pi}{2^{2 m}} \sum_{n=-\infty}^{\infty} e^{i 2 m n \pi N x} \sum_{s=1}^{m} \frac{N^{2 s} \xi_{m-s, m}}{(2 s-1) !}(-1)^{s} 2^{2 s} \\
& \times \sum_{j=0}^{2 s-1}\left(\begin{array}{c}
2 s-1 \\
j
\end{array}\right) \frac{d^{j} A(n \pi N)}{d z^{j}}(-2 i)^{-j} \sum_{k=0}^{q}(-1)^{k}\left(\begin{array}{c}
2 m \\
k
\end{array}\right)(m-m x-k)^{2 s-1-j} \\
&+ 2 \pi i\left[\text { UHP Residues }\left\{A(z) e^{i 2 m z x} \frac{\sin ^{2 m} z-f_{q}(z)}{\sin ^{2 m}(z / N)}\right\}\right. \\
&\text {-LHP Residues } \left.\left\{A(z) e^{i 2 m z x} \frac{f_{q}(z)}{\sin ^{2 m}(z / N)}\right\}\right]
\end{aligned}
$$


Evaluation of the UHP and LHP residue terms of Eqs. (8) requires specification of a particular form of $A(z)$. If we choose the Lorentzian $A(z)=1 /\left(1+\beta^{2} z^{2}\right)$, then

$$
\begin{aligned}
\Psi_{m}^{N}(x)= & \frac{(-1)^{m} \pi}{2^{2 m}} \sum_{n=-\infty}^{\infty} e^{i 2 m n \pi N x} \sum_{s=1}^{m} \frac{N^{2 s} \xi_{m-s, m}}{(2 s-1) !}(-1)^{s} 2^{2 s} \\
& \times \sum_{j=0}^{2 s-1}\left(\begin{array}{c}
2 s-1 \\
j
\end{array}\right) \frac{d^{j} A(n \pi N)}{d z^{j}}(-2 i)^{-j} \sum_{k=0}^{q}(-1)^{k}\left(\begin{array}{c}
2 m \\
k
\end{array}\right)(m-m x-k)^{2 s-1-j} \\
& +\frac{\pi}{\beta} e^{-2 m x / \beta}\left[\frac{\sinh (1 / \beta)}{\sinh (1 /(\beta N))}\right]^{2 m} \\
& -2 \frac{\pi}{\beta}\left(2 \sinh \frac{1}{\beta N}\right)^{-2 m} \sum_{k=0}^{q}(-1)^{k}\left(\begin{array}{c}
2 m \\
k
\end{array}\right) \sinh 2(m-m x-k) / \beta
\end{aligned}
$$

The expressions of Eqs. (8) and $\left(8^{\prime}\right)$ for $\Psi_{m}^{N}(x)$ simplify somewhat and become strictly real when we recall that the coherence function $A(z)$ is an even function of $z$. Again, we note that Eq. (3) prescribes the appropriate value of the integer $q$ in terms of the independent variable $x$. The final result for $\Psi_{m}^{N}(x)$ in Eq. (8) is continuous for all $x$.

III. Second class of integrals. We define a similar and somewhat simpler class of integrals $\Delta_{m}(x)$ as

$$
\Delta_{m}(x)=\int_{-\infty}^{\infty} d z A(z) e^{i 2 m z x} \frac{\sin ^{2 m} z}{z^{2 m}}
$$

$\Delta_{m}$ differs from $\Psi_{m}^{N}$ only in the denominator within the integrand. We continue to restrict $A(z)$ as discussed earlier.

This $\Delta_{m}$ class is easier than the previous case since we do not require a power series expansion of $\left[\sin ^{2 m}(z / N)\right]^{-1}$. We may write, as we did for Eq. (5),

$$
\begin{aligned}
\Delta_{m}(x)= & -2 \pi i \text { Residue }\left\{A(z) e^{i 2 m z x} \frac{f_{q}^{i}(z)}{z^{2 m}}\right\} \\
+ & 2 \pi i\left[\text { UHP Residues }\left\{A(z) e^{i 2 m z x} \frac{\sin ^{2 m} z-f_{q}(z)}{z^{2 m}}\right\}\right. \\
& \text { - LHP Residues } \left.\left\{A(z) e^{i 2 m z x} \frac{f_{q}(z)}{z^{2 m}}\right\}\right] .
\end{aligned}
$$

In this case we have only one singularity on the real axis $(z=0)$. Expanding the quantity $A(z) e^{i 2 m z x} f_{q}(z)$ in a Taylor series about $z=0$ to determine the residue, we 
find

$$
\begin{aligned}
& \Delta_{m}(x)=\frac{\pi}{(2 m-1) !} \sum_{j=0}^{2 m-1}\left(\frac{i}{2}\right)^{j} \frac{d^{j} A(0)}{d z^{j}} \sum_{k=0}^{q}(-1)^{k}\left(\begin{array}{c}
2 m \\
k
\end{array}\right)(m-m x-k)^{2 m-1-j} \\
&+2 \pi i[\text { UHP Residues }\left\{A(z) e^{i 2 m z x} \frac{\sin ^{2 m} z-f_{q}(z)}{z^{2 m}}\right\} \\
&\text { - LHP Residues } \left.\left\{A(z) e^{i 2 m z x} \frac{f_{q}(z)}{z^{2 m}}\right\}\right]
\end{aligned}
$$

Again, evaluation of the UHP and LHP residues awaits our specification of $A(z)$. Choosing the Lorentzian of Eq. $\left(8^{\prime}\right)$, we find

$$
\begin{aligned}
& \Delta_{m}(x)=\frac{\pi}{(2 m-1) !} \sum_{j=0}^{2 m-1}\left(\frac{i}{2}\right)^{j} \frac{d^{j} A(0)}{d z^{j}} \sum_{k=0}^{q}(-1)^{k}\left(\begin{array}{c}
2 m \\
k
\end{array}\right)(m-m x-k)^{2 m-1-j} \\
& +\pi \beta^{2 m-1} e^{-2 m x / \beta}[\sinh (1 / \beta)]^{2 m}-\frac{2 \pi}{\beta}\left(\frac{\beta}{2}\right)^{2 m} \sum_{k=0}^{q}\left(\begin{array}{c}
\left.{ }^{2 m}\right)^{k}\left(\begin{array}{c}
2 m \\
k
\end{array}\right) \sinh 2(m-m x-k) / \beta .
\end{array}\right.
\end{aligned}
$$

The definition of the integer $q$ in Eqs. (11) and $\left(11^{\prime}\right)$ is precisely equivalent to that in Eq. (8). As in the discussion in the previous section, $\Delta_{m}(x)$ is more easily expressed in terms of the residues due to poles of $A(z)$ when $x \geq 1$ or $x \leq-1$. For the $1 /\left(1+\beta^{2} z^{2}\right)$ Lorentzian $A(z)$,

$$
\Delta_{m}(x)=\pi \beta^{2 m-1} e^{-2 m x / \beta}[\sinh (1 / \beta)]^{2 m}, \quad x \geq 1 .
$$

One obtains the result for $x \leq-1$ by replacing $x$ by $-x$ in this equation.

IV. Third class of integrals. We consider another set of integrals $I_{n}(a)$ of the form:

$$
I_{n}(a)=\int_{-\infty}^{\infty} d z \frac{\sin ^{n} z \sin ^{n}(z-a)}{z^{n}(z-a)^{n}} .
$$

As it stands, this integrand has no singularities. We cannot choose a simple contour for integration, however, since the integrand diverges at infinity along the positive and negative imaginary axes. Evaluation of $I_{n}(a)$ is facilitated by a change of variable and application of a trigonometric identity to yield

$$
I_{n}(a)=\int_{-\infty}^{\infty} d z \frac{\left(\cos ^{2} b-\cos ^{2} z\right)^{n}}{(z-b)^{n}(z+b)^{n}} \quad \text { where } \quad b=a / 2 .
$$

The next step requires expansion of the numerator of the integrand of equation (13):

$$
\left(\cos ^{2} b-\cos ^{2} z\right)^{n}=\sum_{m=0}^{n}(-1)^{m}\left(\begin{array}{c}
n \\
m
\end{array}\right) \cos ^{2(n-m)} b \cos ^{2 m} z .
$$

As with the previous integrals, we expand the trigonometric term in terms of imaginary exponentials. In this case,

$$
\cos z=\frac{1}{2}\left(e^{i z}+e^{-i z}\right) .
$$


Raising this form of $\cos z$ to the $2 m$ power, we can classify all terms as bounded along the positive imaginary axis or bounded along the negative imaginary axis. Thus, we partition $\cos ^{2 m} z$ as

$$
\begin{gathered}
\cos ^{2 m} z=\left[\cos ^{2 m} z-f_{m}(z)\right]+f_{m}(z) \\
\text { with } f_{m}(z)=2^{-2 m} \sum_{j=0}^{m-1}\left(\begin{array}{c}
2 m \\
j
\end{array}\right) e^{-i 2 z(m-j)} .
\end{gathered}
$$

We evaluate the integral corresponding to the bracketed term of Eq. (15a) about contour $C_{1}$ and the integral of the remaining $\left(f_{m}(z)\right)$ term about contour $C_{2}$. (Recall the contour definitions of section II.) After performing the summation indicated by Eq. (14), we find that the $\cos ^{2 m} z$ term of Eq. (15a) drops out so that

$$
I_{n}(a)=-2 \pi i \sum_{m=1}^{n}(-1)^{m}\left(\begin{array}{c}
n \\
m
\end{array}\right) \cos ^{2(n-m)} b \sum_{-b,+b} \text { Residue }\left\{\frac{f_{m}(z)}{(z-b)^{n}(z+b)^{n}}\right\}
$$

The summation on $m$ begins at $m=1$, instead of $m=0$, because $f_{0}(z)=0$. (There is no need to partition a constant numerator.) The inner summation of Eq. (16) indicates that we must consider residues at both $z=b$ and $z=-b$.

By expanding $(z+b)^{-n}$ about $z=b$ and multiplying this expansion by a corresponding Taylor series for $f_{m}(z)$, we construct the residue for the $z=b$ pole:

$$
\sum_{q=0}^{n-1}(-1)^{q}(2 b)^{-n-q}\left(\begin{array}{c}
q+n-1 \\
q
\end{array}\right) \frac{1}{(n-1-q) !} \frac{d^{n-1-q} f_{m}(b)}{d z^{n-1-q}} .
$$

Noting that

$$
\frac{d^{s} f_{m}(z)}{d z^{s}}=2^{-2 m} \sum_{j=0}^{m-1}\left(\begin{array}{c}
2 m \\
j
\end{array}\right)[-i 2(m-j)]^{s} e^{-i 2 z(m-j)},
$$

one observes that the residue of the pole at $z=-b$ will be the negative complex conjugate of that at $z=b$. The sum of both residues is then twice the imaginary part of the residue above.

Combining these statements with Eq. (16),

$$
\begin{aligned}
I_{n}(a) & =(-2)^{n+1} \pi a^{-n} \sum_{m=1}^{n}(-1)^{m} 2^{-2 m}\left(\begin{array}{c}
n \\
m
\end{array}\right) \cos ^{2(n-m)} b \\
& \times \operatorname{Im} \sum_{q=0}^{n-1} \frac{i^{n-1-q}}{(2 a)^{2 q}}\left(\begin{array}{c}
q+n-1 \\
q
\end{array}\right) \frac{1}{(n-1-q) !} \sum_{j=0}^{m-1}\left(\begin{array}{c}
2 m \\
j
\end{array}\right)(m-j)^{n-1-q} e^{-i 2 b(m-j)} .
\end{aligned}
$$

In Eq. (17) "Im" stands for "imaginary part". 
We can simplify the equation (17) expression for $I_{n}(a)$ by considering separately the two possibilities of $n$ even and $n$ odd. For $n$ odd (i.e., $n=1,3,5, \ldots$ ),

$$
\begin{aligned}
& I_{n}(a)=\frac{(-1)^{(n+1) / 2} \pi}{a^{n}} 2^{n+1} \sum_{m=1}^{n} \frac{(-1)^{m}}{2^{2 m}}\left(\begin{array}{c}
n \\
m
\end{array}\right)\left(\cos ^{2} \frac{a}{2}\right)^{n-m} \\
& \times\left\{\sum_{q=0}^{(n-1) / 2} \frac{(-1)^{q}}{(2 a)^{2 q}}\left(\begin{array}{c}
2 q+n-1 \\
2 q
\end{array}\right) \frac{1}{(n-1-2 q) !} \sum_{j=0}^{m-1}\left(\begin{array}{c}
2 m \\
j
\end{array}\right)(m-j)^{n-1-2 q} \sin [a(m-j)]\right. \\
& \left.-\sum_{q=1}^{(n-1) / 2} \frac{(-1)^{q}}{(2 a)^{2 q-1}}\left(\begin{array}{c}
2 q+n-2 \\
2 q-2
\end{array}\right) \frac{1}{(n-2 q) !} \sum_{j=0}^{m-1}\left(\begin{array}{c}
2 m \\
j
\end{array}\right)(m-j)^{n-2 q} \cos [a(m-j)]\right\} .
\end{aligned}
$$

For even $n$ (i.e., $n=2,4, \ldots$ ), on the other hand,

$$
\begin{aligned}
& I_{n}(a)=\frac{(-1)^{n / 2} \pi}{a^{n}} 2^{n+1} \sum_{m=1}^{n} \frac{(-1)^{m}}{2^{2 m}}\left(\begin{array}{c}
n \\
m
\end{array}\right)\left(\cos ^{2} \frac{a}{2}\right)^{n-m} \\
& \times\left\{\sum_{q=1}^{n / 2} \frac{(-1)^{q}}{(2 a)^{2 q-1}}\left(\begin{array}{c}
2 q+n-2 \\
2 q-1
\end{array}\right) \frac{1}{(n-2 q) !} \sum_{j=0}^{m-1}\left(\begin{array}{c}
2 m \\
j
\end{array}\right)(m-j)^{n-2 q} \sin [a(m-j)]\right. \\
& \left.-\sum_{q=0}^{n / 2} \frac{(-1)^{q}}{(2 a)^{2 q}}\left(\begin{array}{c}
2 q+n-1 \\
2 q
\end{array}\right) \frac{1}{(n-1-2 q) !} \sum_{j=0}^{m-1}\left(\begin{array}{c}
2 m \\
j
\end{array}\right)(m-j)^{n-1-2 q} \cos [a(m-j)]\right\} .
\end{aligned}
$$

Inserting the specific values of $n=1$ and $n=2$ into Eqs. (18) and (19), respectively, we find

$$
I_{1}(a)=\frac{\pi}{a} \sin a \quad \text { and } \quad I_{2}(a)=\frac{\pi}{a^{2}}\left(1-\frac{\sin 2 a}{2 a}\right) .
$$

Appendix: Physical basis for integrals. The first- and second-order forms of the integrals appearing in the text relate physically to covariance matrix elements which are generated in the mathematical analyses of imaging systems using coherent detection techniques. These applications can include microwave systems relevant to active and/or passive radars, and celestial radio astronomy. For acoustic coherent systems, relations of this type appear in the analysis of both sonar and ultrasonic imaging systems. As mentioned in the introduction, the reader is referred to the work of one of us [1-4] for details of the various applications. Suffice it here to illustrate one example which is not included in the previous work cited.

Consider a multistatic radar (single receive multiple transmitters), where the targets are illuminated in such a manner that the phases of the scattered radiation from the different targets can be treated as statistically independent random variables each of which is uniformly distributed in the interval $(0,2 \pi)$. The scattered signal will be coherently detected by a linear phased array receiver, with the individual antenna elements located at the space points $\mathbf{r}_{n}$. We assume omni-directional individual antenna elements, and narrow band detection of the signal at the microwave wavelength $\lambda$. The coordinate system is chosen so that the polar axis in the spherical polar coordinate system is coincident with the array axis. The scattering space will 
be divided up into elemental volume elements which will be labeled by the index $l$. The beam-formed signal, which leads to focusing at the polar angle $\mu_{\mathrm{foc}}=\cos \left(\theta_{\mathrm{foc}}\right)$, is represented by

$$
E\left(z_{n}, \mu_{\mathrm{foc}}, t\right)=e^{-i k c t} \sum_{l} \frac{\beta_{l}}{R_{l}} e^{i k\left(R_{l}-z_{n}\left(\mu_{l}-\mu_{\mathrm{foc}}\right)\right)} .
$$

Here $\mathbf{k}$ is the carrier wavevector the magnitude of which is given in terms of the carrier frequency $f_{c}$ (Hertz) and the wavelength $\lambda$ as $k=2 \pi f_{c} / c=2 \pi / \lambda ; \boldsymbol{\beta}_{l}$ represents the complex scattering amplitude from the $l$ th volume element. Consider a uniform linear phased array antenna consisting of $N+M+1$ elements which is divided into two overlapping subantennas each of length $(N+1) d$. Here $d$ represents the uniform spacing of the antenna elements. The overlapping elements are common to both subantennas. The signals from the two subantennas are

$$
\mathbf{U}_{1}\left(\mu_{\mathrm{foc}}, t\right)=\sum_{n=-(N+M) / 2}^{(N-M) / 2} E\left(z_{n}, \mu_{\mathrm{foc}}, t\right) ; \quad \mathbf{U}_{2}\left(\mu_{\mathrm{foc}}, t\right)=\sum_{n=(-N+M) / 2}^{(N+M) / 2} E\left(z_{n}, \mu_{\mathrm{foc}}, t\right) .
$$

The correlation between the signals received in these two antennas is expressed by the off-diagonal covariance matrix elements,

$$
\left.\Gamma_{1,2}\left(\mu_{\mathrm{foc}}, t\right)=\left\langle\mathbf{U}_{1}\left(\mu_{\mathrm{foc}}, t\right)\right) \mathbf{U}_{2}^{*}\left(\mu_{\mathrm{foc}}, t\right)\right\rangle .
$$

Here the brackets represent an average taken over an ensemble of scatterers. Due to the statistical independence of the complex scattering amplitudes, only the coherent part of the scattering gives a nonzero contribution to the ensemble average,

$$
\left\langle\beta_{l} \beta_{l^{\prime}}^{*}\right\rangle=\sigma_{l} \delta_{l l^{\prime}}
$$

Accordingly,

$$
\Gamma_{1,2}\left(\mu_{\mathrm{foc}}, t\right)=\sum_{l} \frac{\sigma_{l}}{\left(4 \pi R_{l}\right)^{2}} e^{-i 2 \pi\left(\mu_{l}-\mu_{\mathrm{foc}}\right) M d / \lambda} \frac{\sin ^{2}\left[\pi(N+1)\left(\mu_{l}-\mu_{\mathrm{foc}}\right) d / \lambda\right]}{\sin ^{2}\left[\pi\left(\mu_{l}-\mu_{\mathrm{foc}}\right) d / \lambda\right]} .
$$

The sum over $l$ is now converted into a volume integral in spherical polar coordinates,

$$
\sum_{l} \sigma_{l} G\left(\mu_{l}\right) \rightarrow \int_{0}^{\infty} R^{2} d R \int_{0}^{2 \pi} d \phi \int_{-1}^{+1} d \mu \sigma(R, \phi, \mu) G(\mu) .
$$

Here $G\left(\mu_{l}\right)$ is a general function of the polar angle variable. Using these results, with the normalized covariance matrix elements defined as

$$
\gamma_{1,2}\left(\mu_{\mathrm{foc}}=0\right)=\frac{\Gamma_{1,2}}{\sqrt{\Gamma_{1,1} \Gamma_{2,2}}},
$$

we obtain

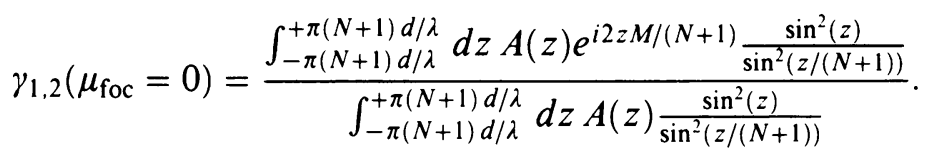

For practical phased array systems, the total phased array antenna length, $(N+1) d$, must be long compared to the wavelength. This limit coupled with the fact that 
real apodizing functions converge rapidly to zero at large polar angles due to the beam patterns of the individual receiving antenna elements, permits us to extend the integration limits in the above expressions to $\pm \infty$. The resulting integral expression with the limits extended to $\pm \infty$ is one of the forms considered in the text.

\section{REFERENCES}

[1] S. D. Silverstein, Coherence and speckle reduction in compounded correlated phased arrays: Synthetic aperture radar, J. Opt. Soc. Amer. A, vol. 3, 1925-1934 (Nov. 1986)

[2] S. D. Silverstein and M. O'Donnell, Speckle reduction using correlated mixed-integration techniques, Proc. SPIE Int. Symp. on Pattern Recog. and Acoustic Imaging, Vol. 768, 168-172 (Feb. 1987)

[3] M. O'Donnell and S. D. Silverstein, Optimum displacement for compound image generation in medical ultrasound, to be published in IEEE Trans. on Ultrasonics, Ferroelectrics, and Frequency Control (1988)

[4] S. D. Silverstein and M. O'Donnell, Theory of frequency and temporal compounding in coherent imaging. Speckle suppression and image resolution, J. Opt. Soc. Amer. A, vol. 5, 101-113 (January 1988)

[5] G. F. Carrier, M. Krook, and C. E. Pearson, Functions of a complex variable, McGraw-Hill, New York, 1966

[6] G. Arfken, Mathematical methods for physicists, Academic Press, New York, 1970 